the inflation, deflation or artificial respiration.

On condition that the mean posterior fossa volume should be $13 \mathrm{~g}$, and unless it developed no farther than the stage III, about 15 volume percent of posterior fossa mass might be hopeful.

\title{
7. Hydrostatic Brain Edema Caused by Vasomotor-Paresis
}

\author{
Katsuzro Matsumoto, Kiyoshi Iwatsuki Akira Matsumoto, \\ Norihiko Mizukawa Shogo Nagao, Taketoshi Marabe, \\ Kenzi Sujuki, Hiromasa Nakayama, Hiroo Oota \\ and Akira Nishimoto \\ Department of Neurosurgery, University of Okayama
}

\section{Cerebral Hemodynamics and Gerebrovascular Reactivity in Experimental Subarachnoid Hemorrhage}

\author{
Takao Ito, Yoshitoki Murase, Sengai Tanaka, \\ Hiromu Yamada, Kazuki Sakata and Takao Taketomo \\ 2nd Department of Surgery, Gifu University \\ School of Medicine
}

Purpose: Experimental subrachnoid hemorrhage was produced and consequent spasm of cerebral arteries, including Willis' circle and its main branches, was confirmed angiographically. This phenomenon was observed from the standpoints of cerebral circulation and cerebrovascular reactivity.

Method: In adult mongreal dogs which were maintained under artificial respiration, degree of the arterial spasm was sequentially observed angiographically and changes in cerebral blood flow were measured with an electromagnetic flow meter. Cerebrospinal fluid pressure was measured in the cisterna magna and systemic arterial blood pressure was measured at the abdominal aorta. Experimental subarachnoid hemorrhage was produced by injecting $2 \mathrm{ml}$ of autogenous blood into the chiasmal cistern according to McQueen's method. Effect of inhalation of $5 \% \mathrm{CO}_{2}$ in air on cerebral blood flow in the presence of arterial spasm was observed. Observation was also made on cerebrovascular reactivity. Under hypertension produced either by noradrenalin or adrenalin administration or by clamping the aortic arch, and under hypotension produced by exsanguination. 